\title{
ANALYSIS OF TRANSVERSE BEAM OSCILLATION AT PHOTON FACTORY
}

\author{
Weixing Cheng, Takashi Obina \\ KEK, Oho 1-1 Tsukuba, Ibaraki ken, Japan
}

\section{Abstract}

At KEK Photon Factory, FPGA based bunch by bunch feedback system to cure the transverse instabilities has been operating stably since Oct. 2005. Specification and performance of the system will be introduced here. Up to $640 \mathrm{k}$ turns data can be stored in the buffer for each bunches, transient measurement has been done to analyze the instability modes under different beam conditions, which helps to understand the instability sources. A new generation digital BPM electronics (Libera) has also been evaluated at Photon Factory, which can give the beam position data at different bandwidth simultaneously. Bunch by bunch beam oscillation, together with the digital turn-by-turn beam position measurement, injection oscillation damping is recorded and analyzed, transverse beam oscillation with and without the bunch by bunch feedback system will be shown in this paper. With turn by turn beam position data, many machine physics parameters can been measured, one example is the précis tune measurement; centre of mass of the bunched beam can been plotted in phase space, from which the nonlinear beam dynamics can be revealed.

\section{INTRODUCTION}

Transverse bunch-by-bunch feedback system has been in operation since Oct. 2005, both horizontal and vertical betatron oscillation are suppressed well in one feedback control loop. Detailed description can been found at the former papers ${ }^{[1,2]}$. Table 1 lists the specification of this system. Well adjusted two BPM buttons differential signal, which include the position information of each bunch, get sampled by six 12-bit ADCs. Sampling clock is fixed to one sixth of the ring RF frequency, that makes sure every bunch can be sampled. FIR filter cut the DC components as well as revolution harmonics, only betatron component with additional phase shift pass it to DAC output. Two high power wide band amplifiers drive the $45 \mathrm{~cm}$ stripline kicker to give the corresponding force for individual bunches. ADC samples can been upgraded to a circular buffer, which have a total length of $32 \mathrm{M}$ for each ADC, that's corresponding to $640 \mathrm{k}$ turns $(\sim 400 \mathrm{~ms})$ data for photon factory ring.

Transient measurement turn off the bunch-by-bunch feedback loop for some time and turn on again, with such a long history buffer, all the bunches oscillation can been recorded during this $\mathrm{ON}->\mathrm{OFF}->\mathrm{ON}$ procedure. FFT of the time domain bunch position information gives the unstable modes, growth/damping time can also be fitted. In PF's normal multi-bunch user operation, the beam is not lost even without feedback, it's interesting to turn off the feedback loop for quite long time $(\sim 100 \mathrm{~ms})$ to get the habit of bunches.

Table 1: Main parameters of PF transverse bunch-bybunch feedback system

\begin{tabular}{ll}
\hline RF frequency & $500.1 \mathrm{MHz}$ \\
Harmonic Number & 312 \\
$\mathrm{f}_{\mathrm{rev}}$ & $1.6029 \mathrm{MHz}$ \\
Tune $\nu_{\mathrm{x}} / \nu_{\mathrm{y}} / v_{\mathrm{s}}$ & $9.60 / 5.28 / 0.014$ \\
Damping time $\tau_{\mathrm{x}} / \tau_{\mathrm{y}} / \tau_{\mathrm{s}}$ & $7.8 / 7.8 / 3.9 \mathrm{~ms}$ \\
ADCs & $12 \mathrm{bit}, 6 \mathrm{Channels}$ \\
ADC Clock & $83.35 \mathrm{MHz}=\mathrm{f}_{\mathrm{RF}} / 6$ \\
FIR & 20 tap \\
DAC Clock & $500.1 \mathrm{MHz}$ \\
Buffer length & $32 \mathrm{M}$ for each ADC \\
& $\sim 640 \mathrm{k} \mathrm{turns}$ \\
& $\sim 400 \mathrm{~ms}$ \\
\hline
\end{tabular}

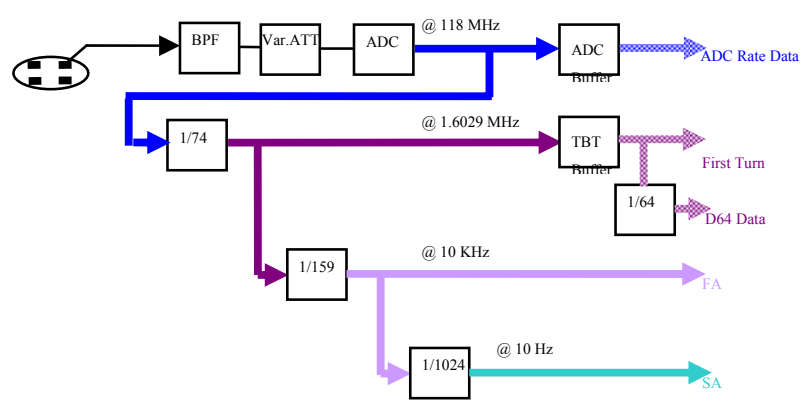

Figure 1. Data flow for PF Libera electronics

New generation BPM electronics called Libera ${ }^{[3]}$ has been tested at photon factory. In Libera, the 4 channel direct BPM button signals pass through the band pass filter $\left(\mathrm{f}_{\text {center }}=500.1 \mathrm{MHz}, \mathrm{BW}=10 \mathrm{MHz}\right)$, two programmable attenuators $(0-31 \mathrm{~dB}$ each) can adjust the signal level to suitable value. Band pass filtered signal get sampled around $118 \mathrm{MHz}$, which is $74^{\text {th }}$ harmonic of PF ring revolution frequency, $\mathrm{ADC}$ sampled high rate data get decimated and filtered inside FPGA to get turn-byturn data rate $(\sim 1.6029 \mathrm{MHz})$. More decimation and filtered are implemented in the FPGA to get FA rate data for fast orbit feedback, SA rate data for COD display or slow orbit feedback. Figure 1 shows the data flow inside 
the PF Libera electronics. With turn-by-turn beam position data, machine parameters can be revealed from that, for example, betatron tune, injection damping and beam centre of mass oscillation during injection, these information are all very helpful for machine tuning. Synchronize with transverse feedback transient measurement, turn-by-turn data also gives the mode 0 growth/damping plot, which is the dominant unstable mode due to ion-trapping and resistive-wall instability in $\mathrm{PF}$ ring.

\section{TRANSIENT MEASUREMENT}

Timing for the transient measurement are shown in Fig. 2 , $\mathrm{CH} 4$ is the memory update trigger signal, $\mathrm{CH} 3$ is feedback ON/OFF control signal, while $\mathrm{CH} 2$ gives the DAC output and $\mathrm{CH} 1$ is the beam oscillation. While the feedback turns off, DAC has no output, beam getting unstable and large beam oscillation appears on the oscilloscope. Depends on the beam current, filling pattern and vacuum condition, the unstable growth time might be very long. While the feedback turns ON, DAC output has sharp peak to suppress the large oscillation, the damping time is less than $1 \mathrm{~ms}$.

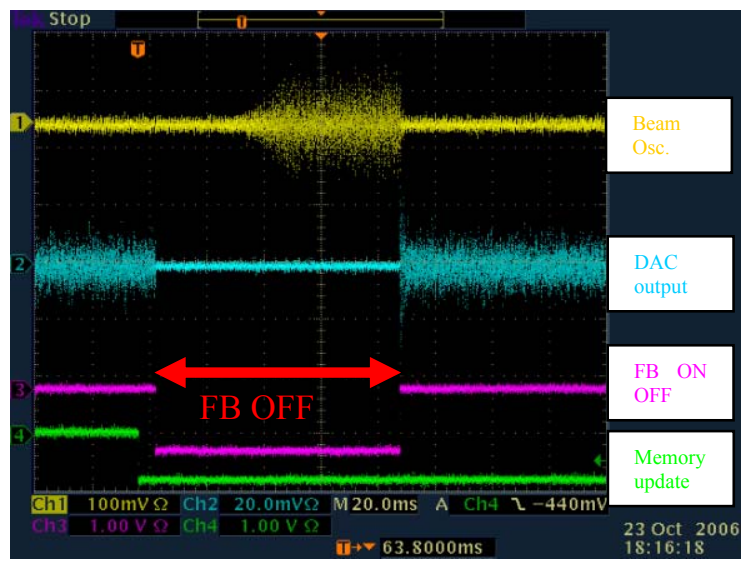

Figure 2. Timing of PF transient measurement

To get the unstable modes specification, FFT calculation is applied for the ADC time-domain position data, FFT window size and step size can be selected to get suitable resolution. Since not all the buckets are filled for normal running, it's convenient to use "projecting" mode number, that is to say even there has 280 bunches which corresponding to 280 modes in frequency-domain, we use 312 modes to compare with the real spectrum. Figure 3 gives one of the results, 3-D plot of all the horizontal modes, the most strong one while feedback OFF is mode0 and mode-7, this has been confirmed with spectrum analyzer. The mode-7 horizontal sideband arise after $\sim 25$ ms later than mode- 0 , while the mode- 7 betatron sideband getting stronger, the mode- 0 betatron sideband decreasing, it seems some of the beam oscillation energy is exchanging between these two modes. While the beam current is less than $350 \mathrm{~mA}$, mode-7 sideband never appears. We believe that mode- 7 is coming from ion- trapping instabilities although the mechanism is not clear, while mode- 0 should be contributed from ions and resistive wall instability.
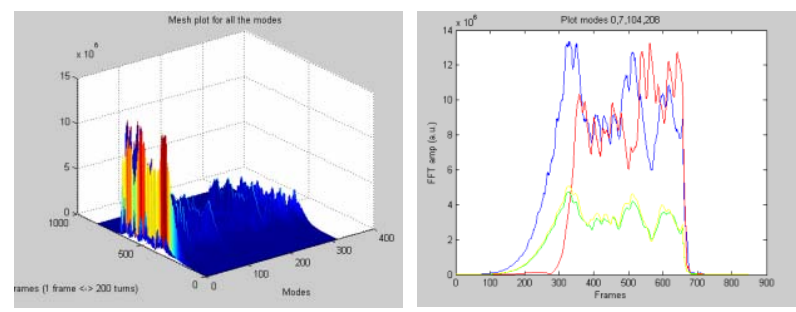

Figure 3. Unstable modes plot

As mode- 0 is the dominant unstable mode in PF ring, fitting the growth part of this mode gives the growth rate (growth time). There has other methods to measure the growth time is using the turn-by-turn data from Libera, send the transient measurement trigger signal to Libera and we can fit the FFT spectrum of turn-by-turn data, this can gives the growth/damping information of mode- 0 only. Another easiest way to estimate the growth time roughly is using a spectrum analyzer, set the frequency to betatron frequency which interested and use "zero SPAN" function, an external transient measurement trigger signal should be sent to the spectrum analyzer.
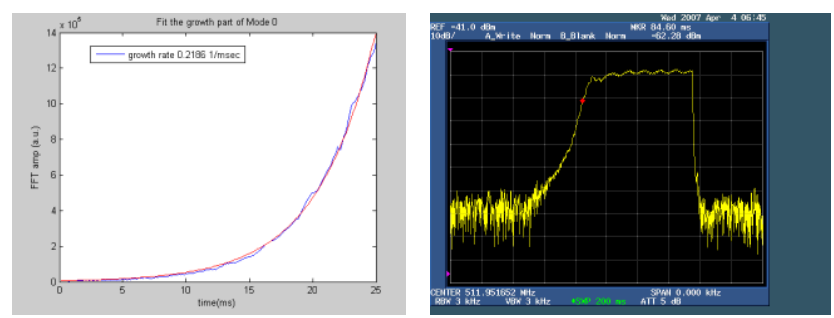

Growth time vs. Average vacuum pressure

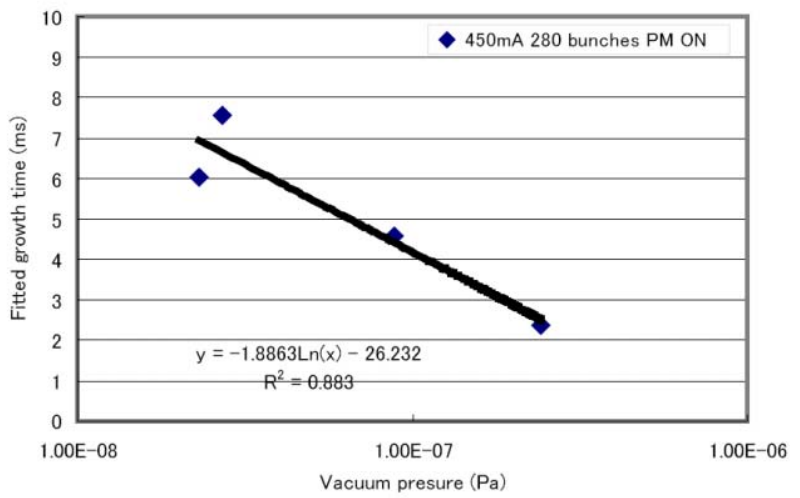

Figure 4. Growth time measurement, 1) Upper-left, fitting the growth part of mode 0 from ADC data; 2) upper-right, estimate the growth time using spectrum analyzer; 3) lower, growth time at different vacuum condition.

Unstable mode growth time is heavily depends on the filling pattern, the vacuum pressure and RF phase modulation status. RF phase modulation around $2 \mathrm{fs}$ is used to suppress the longitudinal instabilities as well as beam lifetime increasing, with RF phase modulation ON 
and OFF, the transverse unstable mode growth time is different. Figure 4 shows the fitting growth time from ADC data and spectrum analyzer "zero SPAN" plot during one transient measurement, the lower curve gives the growth time at different vacuum pressure, which proves ion-trapping is one of the main sources. As the vacuum condition getting better, the unstable mode 0 growth time getting longer.

There has only horizontal instabilities, no vertical unstable mode observed even with the insertion devices closed after the straight-section upgrade in PF ring, which seems quite strange. Insertion device has very small gap in vertical plane, resistive-wall instability should be strong, especially for mode-0, however, only strong horizontal betatron sideband observed for mode-0. More machine studies should be carried on to explain it.

\section{TURN-BY-TURN MEASUREMENT}

Libera electronics has been tested at PF ring, for the low rate COD data, more machine studies are going on to compare with the existing BPM systems to get a high accurate beam position information. With turn-by-turn data, Tune block diagram with about $1 \mathrm{~Hz}$ update rate, while tune changed, we can see the history trajectory and determine whether it passes some dangerous resonant lines. Timing domain plot of the transverse center-ofmass oscillation gives the influence by the injection kicker bump, for top-up injection, this influence to the whole ring orbit should be tuned as small as possible. Figure 5 gives a example at different injection kicker angle settings, a tune block diagram during injection tuning presented too.
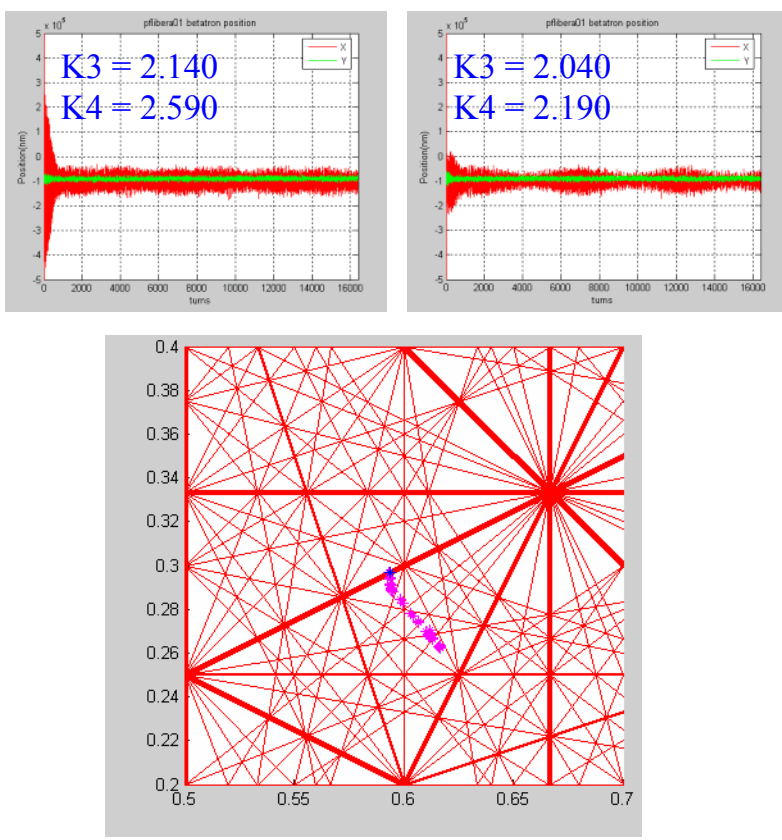

Figure 5. Transverse oscillation and tune plot during injection tuning.
Figure 6 gives the machine study result while the vertical fractional tune is set around 0.25 , which lead to $4^{\text {th }}$ order resonance.

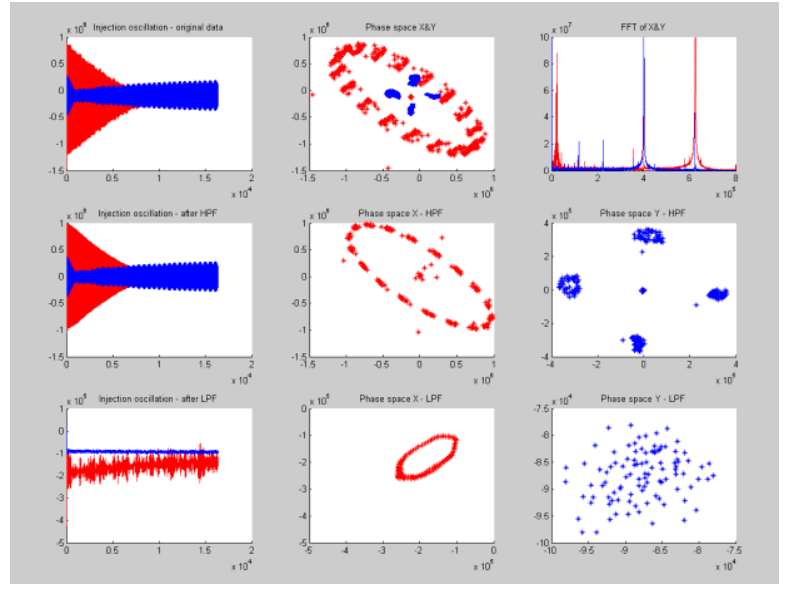

Figure 6. Fourth order resonance for vertical betatron oscillation.

\section{SUMMARY}

Transverse oscillation at $\mathrm{PF}$ ring has been analyzed using bunch-by-bunch feedback ADC data. Growth time at different beam current, different vacuum condition and filling gap has been compared, which confirms the iontrapping instability exist, mode 7 for the horizontal betatron oscillation also appears, the mechanism of that is not so clear.

Turn-by-turn BPM measurement gives another method to understand the beam oscillation character, these information proves to be helpful for the machine tuning and other advanced studies.

The authors thank to staff of the PF light source division for support of the machine studies.

\section{REFERENCES}

[1] Bunch-by-bunch Feedback for the Photon Factory Storage Ring, W.X.Cheng, T.Obina, T.Honda et. al. EPAC2006

[2] Single-loop Two-dimensional Transverse Feedback for Photon Factory, T.Nakamura, K.Kobayashi, W.Cheng et. al. EPAC2006

[3] http://www.i-tech.si/ 